analysis, tumour size $>2 \mathrm{~cm}$ (odds ratio: 49.1, 95\% CI: 1.2 to $1990.1, \mathrm{p}=0.04$ ) and irregular border (odds ratio: 27.5, 95\% CI: 1.0 to $747.0, \mathrm{p}=0.05$ ) were independent factors of malignant GISTs.

Conclusions Tumour size $>2 \mathrm{~cm}$ and irregular border on EUS can be used to predict the malignant gastric GISTs.

\section{IDDF2018-ABS-0218 EFFICACY OF FAECAL MICROBIOTA THERAPY IN PATIENTS WITH STEROID DEPENDENT ACTIVE ULCERATIVE COLITIS}

Ramit Mahajan*, Vandana Midha, Varun Mehta, Arshdeep Singh, Husanpreet Khattar, Yogesh Gupta, Vikram Narang, Ajit Sood. Dayanand Medical College and Hospital, India

\subsection{6/gutjnl-2018-IDDFabstracts. 161}

Background Faecal microbiota transplantation (FMT) has been shown to be effective in active ulcerative colitis (UC) by targeting gut dysbiosis. We assessed the role of FMT in steroiddependent UC patients.

Methods In this trial, patients with steroid-dependent active UC were treated with FMT using random unrelated donors, by the colonoscopic approach, at weeks $0,2,6,10,14,18$ and 22. Patients with steroid-dependent UC treated who were treated without FMT in past, with azathioprine as a steroidsparing agent were taken as historical controls. The primary outcome was the achievement of steroid-free clinical remission (Mayo score $=30 \%$ and $\geq 3$ points compared to baseline) and endoscopic remission (Mayo score 0 or 1). $16 \mathrm{~s}$ rRNA gene sequencing was done for analysing changes in microbial composition after FMT.

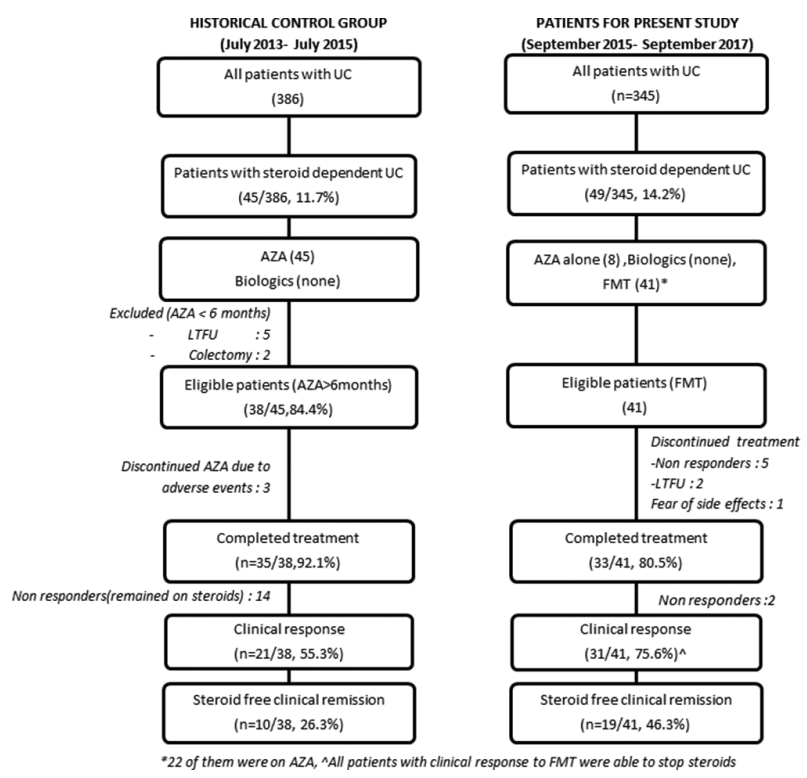

Abstract IDDF2018-ABS-0218 Figure 1 Patient flow diagram uc ulcerative colitis aza azathioprine fmt faecal microbiota transplantation

Results Between September 2015 - September 2017, 41 patients with steroid-dependent UC underwent FMT, 33 completed seven sessions over 22 weeks while 8 discontinued treatment (non-response: 5, lost to follow up: 2, fear of side effects: 1) (figure 1). At week 22, the primary endpoint (steroid-free clinical remission) was achieved in 46.3\% (19/41) patients treated with FMT compared to $26.3 \%$ (10/38) in historical controls treated with azathioprine $(p=0.065)$. Clinical response $(31 / 41,75.6 \%)$ and endoscopic remission $(26 / 41$, $63.4 \%$ ) with FMT were significantly higher than controls $(55.3 \%$ and $39.5 \%$ respectively, $\mathrm{p}=0.005)$ (IDDF2018-ABS0218 Figure 2). Adverse events necessitating discontinuation were noted in $3 / 38$ (7.89\%) controls treated with azathioprine, but not with FMT.

Conclusions A multi-session FMT by a colonoscopic route is a promising therapeutic option for steroid-dependent UC patients, as it induces clinical remission and withdrawal of steroids in $46.3 \%$ and $75.6 \%$ patients respectively.

\section{IDDF2018-ABS-0221 MAINTENANCE WITH FAECAL MICROBIOTA TRANSPLANTATION ENHANCES DEEP REMISSION IN PATIENTS WITH ULCERATIVE COLITIS IN CLINICAL REMISSION}

Vandana Midha*, Arshdeep Singh, Ramit Mahajan, Varun Mehta, Husanpreet Khattar, Vikram Narang, Ajit Sood. Dayanand Medical College and Hospital, India

\subsection{6/gutjnl-2018-IDDFabstracts. 162}

Background Faecal microbiota transplantation (FMT) is beneficial in patients with active ulcerative colitis (UC), but its role in the maintenance of clinical remission and mucosal healing has not been assessed.

Methods This was a prospective, double-blind, randomised placebo-controlled trial conducted at Dayanand Medical College and Hospital, India. Twenty-eight patients with UC in clinical remission (Mayo Score $=1$ ) and histological remission (Nancy grade 0,1$)$ at 48 weeks.

Results Twenty-eight patients (50\% males, aged 37.21 \pm 15.25 years) with UC in clinical remission were randomised to groups A and B. At the end of 48 weeks, none of the patients in either group suffered a clinical relapse $(p=1)$. Deep remission was noted in significantly higher number of patients with FMT $(\mathrm{n}=12,85.71 \%)$ when compared with placebo $(\mathrm{n}=4,28.57 \%) \quad(\mathrm{p}=0.002)$. Histological remission was twice as high in patients treated with FMT $(n=8,57.14 \%$ vs placebo, $n=4,28.57 \% ; \mathrm{p}=0.13)$. Rate of endoscopic response was also higher with FMT $(n=8,57.14 \%)$ when compared to placebo $(n=0$, zero\% $)(p=0.001)$. No serious adverse events were noted.

Conclusions Maintenance with FMT can enhance achievement of deep remission and histological remission in patients with $\mathrm{UC}$ in clinical remission.

\section{IDDF2018-ABS-0222 USE OF MAGNETIC RESONANCE IMAGING TO EVALUATE THE EFFECTIVENESS OF TREATMENT FOR PERIANAL FISTULIZING CROHN'S DISEASE}

${ }^{1}$ Xiaohan Yan*, ${ }^{2}$ Qi Feng, ${ }^{2}$ Yunqi Yan, ${ }^{1}$ Xitao Xu, ${ }^{1}$ Mingming Zhu. ${ }^{1}$ Division of Gastroenterology and Hepatology, Key Laboratory of Gastroenterology and Hepatology, Ministry of Health; Shanghai Inflammatory Bowel Disease Research Center; Renji Hospital, School of Medicine, Shanghai Jiao Tong University; Shanghai Institute of Digestive Disease, China; 'Department of Radiology, Renji Hospital, School of Medicine, Shanghai Jiao Tong University, Shanghai, China

\subsection{6/gutjnl-2018-IDDFabstracts. 163}

Background Data on the radiologic evaluation of perianal fistulizing Crohn's disease (PFCD) naive to anti-tumour necrosis 
factor therapy are scarce, especially in Asian populations. We assessed the effectiveness of infliximab (IFX) and azathioprine on PFCD and explored predictors of 'deep remission' based on clinical and radiologic assessments.

Methods Patients with Crohn's disease and active anal fistulas attending our centre for IFX therapy were prospectively enrolled. Each patient underwent clinical examination according to the Fistula Drainage Assessment Index, magnetic resonance imaging (MRI) to determine Van Assche score, $\mathrm{Ng}$ score, and main fistula length, endoscopy, assessment of Crohn's disease activity index (CDAI) and perianal Crohn's disease activity index (PCDAI), and laboratory tests up to 2 weeks prior to the start of and up to 2 weeks after the sixth IFX therapy (Week 32). Patients with PFCD treated with other medicines such as FK 506 or mesalazine and evaluated by two MRIs were retrospectively enrolled.

Results Of 38 patients treated with IFX, 52.6\% achieved clinical remission, and $42.1 \%$ achieved deep remission. The only predictor of deep remission was simple fistula $(\mathrm{p}=0.004$, odds ratio $=3.802,95 \%$ confidence interval: 1.541-9.383). Van Assche score (from $14.5 \pm 4.26$ to $7.36 \pm 7.53$; figure 1 ), CDAI (from $170 \pm 92$ to $71 \pm 69$ ), and PCDAI (from $7.45 \pm 2.65$ to $2.44 \pm 3.2$ ) decreased significantly after six IFX treatments. Our findings suggest that Van Assche score has some limitations related to its inability to reflect new fistulas and abscesses in some circumstances. We used $\mathrm{Ng}$ score to compare the changes between the initial and follow-up MRIs in each patient, which is more objective and suitable for monitoring the therapeutic effect.

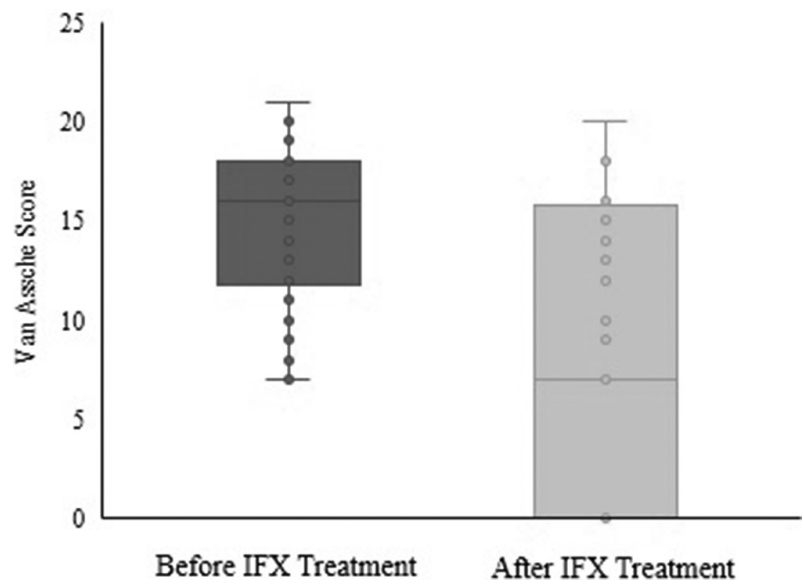

Abstract IDDF2018-ABS-0222 Figure 1 Van assche scores were significantly reduced after six infliximab treatments
Conclusions IFX is effective for the treatment of PFCD. MRI is the gold standard for evaluating PFCD, but Van Assche score has some limitations.

\section{IDDF2018-ABS-0223 AN EASTERN CHINESE MULTICENTER SURVEY ON HEALTH-RELATED QUALITY OF LIFE IN INFLAMMATORY BOWEL DISEASE PATIENTS AND DISEASE-RELATED PERCEPTION OF CAREGIVERS}

${ }^{1}$ Chenwen Cai*, ${ }^{2}$ Naizhong Hu, ${ }^{3}$ Yihong Fan, ${ }^{4}$ Chengdang Wang, ${ }^{5}$ Chunxiao Chen, ${ }^{1}$ Jun Shen, ${ }^{1}$ Meilan Huang, ${ }^{1}$ Di Zhao, 'Lijie Lai, ${ }^{1}$ Tianrong Wang, ${ }^{1}$ Yuqi Qiao, ${ }^{1}$ Qing Zheng, 'Zhihua Ran. 'Division of Gastroenterology and Hepatology, Renji Hospital, School of Medicine, Shanghai Jiao Tong University; Shanghai Inflammatory Bowel Disease Research Center, Shanghai, China; ${ }^{2}$ Department of Gastroenterology, The First Affiliated Hospital of Anhui Medical University, Hefei, Anhui Province, China; ${ }^{3}$ Department of Gastroenterology, The First Affiliated Hospital of Zhejiang Chinese Medical University, Hangzhou, Zhejiang Province, China; ${ }^{4}$ Department of Gastroenterology, The First Affiliated Hospital of Fujian Medical University, Fuzhou, Fujian Province, China; ${ }^{5}$ Department of Gastroenterology, The First Affiliated Hospital, Medical College of Zhejiang University, Hangzhou, Zhejiang Province, China

\subsection{6/gutjnl-2018-IDDFabstracts. 164}

Background Inflammatory bowel disease (IBD) may impair patients' health-related quality of life (HRQOL) and impose burdens on caregivers. We aim to survey HRQOL including demographic characteristics of IBD patients and to evaluate the diseaserelated perception of their caregivers in East China.

Methods Patients above 18 years old with established Crohn's disease (CD) or ulcerative colitis (UC) from 5 medical centres in East China between December 2016 and July 2017 were enrolled. Patients' detailed demographic and clinical information were documented. Demographic data of the investigated regions were extracted from China Population and Employment Statistics Yearbook 2016 for comparison. Patients HRQOL was assessed by short IBD questionnaire (SIBDQ) and patient-reported $0-10$ score (IBD-10). The major caregiver of each patient was surveyed by IBD-10 to estimate patient's condition from caregiver's viewpoint.

Results A total of 601 IBD patients were included in this study. The proportion of post-secondary school education $(51.4 \%$ vs $28.1 \%, \mathrm{p}<0.0001)$ and the unemployment rate $(31.8 \%$ vs $2.9 \%-4.0 \%, \mathrm{p}<0.0001)$ was significantly higher in IBD cohort than in general population. Patients in active phase had remarkably lower SIBDQ and IBD-10 scores than those in remission, meanwhile, the scores declined along with the illness severity (table 1). Long disease duration, illness activity, unemployment and corticosteroid treatment were risk factors for poor HRQOL while high BMI provided a
A

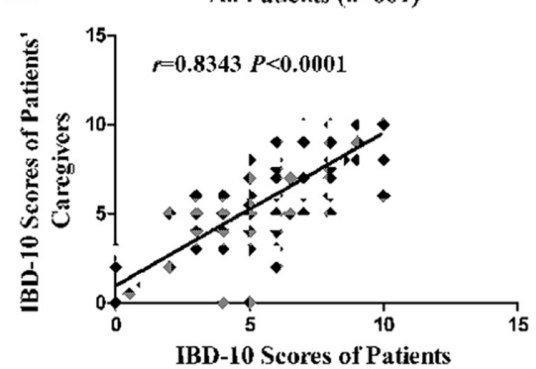

B

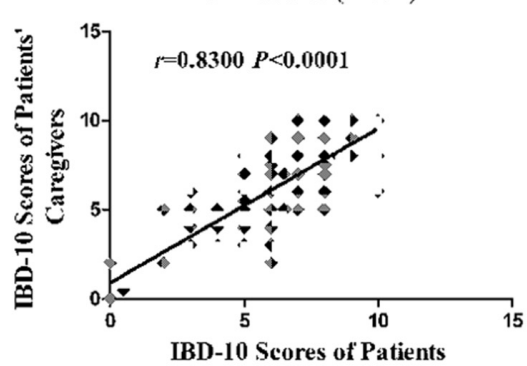

C

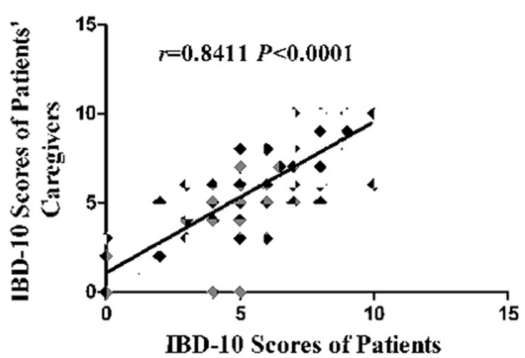

Abstract IDDF2018-ABS-0223 Figure 1 Correlation between ibd 10 scores of ibd patients and their caregivers 